\title{
EDITORIAL
}

\section{Pulmonary thromboendarterectomy almost 50 years after the first surgical attempts}

\section{Cerveri, A M D'Armini, M Viganò}

Heart 2003;89:369-370

With increased experience in preoperative evaluation, surgical approach and postoperative care of chronic thromboembolic pulmonary hypertension, pulmonary thromboendarterectomy can be performed with an acceptably low risk of death. Most patients, even those in a very compromised state, have excellent, long lasting results

See end of article for authors' affiliations

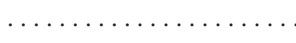

Correspondence to: Dr Isa Cerveri, Clinica Malattie Apparato Respiratorio, Via Taramelli 5, 27100 Pavia, Italy; i.cerveri@libero.it

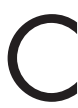
hronic thromboembolic obstruction of the major pulmonary arteries is a potential long term consequence of acute pulmonary embolism. ${ }^{1}$ The actual prevalence of chronic thromboembolic pulmonary hypertension (CTEPH) is almost certainly underestimated. ${ }^{2}$ It is the only type of pulmonary hypertension that can be successfully treated with conservative surgery-that is, pulmonary thromboendarterectomy (PTE) - in selected patients. ${ }^{2-4}$ CTEPH of sufficient severity to warrant surgical intervention is much more common than previously suspected. Each year there are 500-2500 patients with this condition in the USA, accounting for $0.1-0.5 \%$ of patients with pulmonary emboli who survive. ${ }^{3}$

The PTE operation, as first performed at the University of California, San Diego, ${ }^{5}$ is now being systematically employed in about 10 centres around the world. Despite this, we concur with Jamieson's editorial, published four years ago, which stated: "many physicians are unaware that a corrective operation exists, and rely instead on referral for transplantation or merely palliative medical treatment". ${ }^{2}$ There is need for a widespread increase in awareness of both the prevalence of this condition and the possibility of a conservative surgical cure.

PTE can be successfully performed in selected centres with a multidisciplinary approach involving the specialities of surgery, pulmonary medicine, critical care, cardiology, anaesthesiology, and radiology. ${ }^{3}$ In our experience, having an active lung transplant programme in the same centre with the same surgeons is a further, substantial advantage, as patients affected by СTPH can be offered all possible options and, in the case of unsuccessful PTE, an effective surgical alternative.$^{67}$ With this approach, PTE can be performed with an acceptably low risk of death. Overall, the perioperative mortality rate in our centre is $10 \%(8 / 80)$; four patients died of massive alveolar haemorrhage in the immediate postoperative period-one died from severe haemoptysis three days after PTE, and three died from acute right ventricular insufficiency.
PTE promptly reduces pulmonary hypertension and restores cardiac output, producing an excellent symptomatic improvement in almost all patients, even those who were very compromised. $^{2-7}$

There is complete agreement on the short term results of PTE on clinical and cardiopulmonary function status while, at least in the initial reports, there were some discrepancies concerning long term results. The more recent studies that have addressed the long term effects of PTE in large series of patients agree that the beneficial effects persist for many years after the operation in the majority of patients. ${ }^{48}$

An alternative interventional strategy of balloon pulmonary angioplasty for patients in whom surgery is not feasible has recently been implemented.' This is a sufficiently promising technique that warrants randomised comparison with medical treatment in CTEPH patients with surgically inaccessible disease or comorbid illness.

\section{SURGICAL APPROACH AND EARLY POSTOPERATIVE COURSE}

The selection criteria for surgery are currently well standardised. ${ }^{3}$ Briefly, in our centre the selection is based on a combination of clinical (only patients in New York Heart Association (NYHA) functional class III or IV), anatomical (lesions at the level of the main, lobar and segmental pulmonary branches), and haemodynamic (pulmonary vascular resistance (PVR) $>300$ dynes ${ }^{*} \mathrm{sec}^{*} \mathrm{~cm}^{-5}$ ) characteristics. PTE is postponed for patients with a fair clinical status (NYHA class II) and an acceptable haemodynamic profile (PVR $<300$ dynes ${ }^{*} \mathrm{sec}^{*} \mathrm{Cm}^{-5}$ caused by a still elevated cardiac output), while patients with exclusively distal lesions (segmental or subsegmental pulmonary branches) are excluded and should be considered for double lung transplantation. ${ }^{6}$

The location and extent of the proximal thromboembolic obstruction are the most critical determinants of operability. In terms of the extent of obstruction, the anatomical and haemodynamic findings must be interpreted in concert. This determination is crucial: if the haemodynamic impairment derives from mainly surgically inaccessible disease or from the resistance conferred by a secondary, small vessel arteriopathy,

Abbreviations: $\mathrm{CTEPH}$, chronic thromboembolic pulmonary hypertension; NYHA, New York Heart Association; PTE, pulmonary thromboendarterectomy; PVR, pulmonary vascular resistance 
then pulmonary hypertension will persist postoperatively and may have adverse short term and long term effects. ${ }^{3}$ A central focus of the evaluation process therefore involves integrating the haemodynamic, angiographic, and angioscopic findings as a means of separating the central, surgically accessible component of the haemodynamic impairment from that which is not surgically correctable.

The current surgical approaches have been reviewed at length in several recent publications. ${ }^{10}{ }^{11}$ Considerable experience is required to identify and establish the correct plane of dissection. Modifications of the surgical approach, intended to decrease risk or improve haemodynamic outcome, continue to be explored. The surgical team in our centre, unlike the San Diego group at the University of California, usually neither clamps the ascending aorta nor uses cardioplegia because we have found that similar cardiac protection is achieved by perfusing the fibrillating heart with cold blood while the left ventricle is vented through the right superior pulmonary vein. ${ }^{6}$

Careful postoperative management is essential to the success of the operation. Besides the problems encountered in the postoperative period that are shared by patients undergoing other forms of cardiac surgery, the postoperative care of patients undergoing PTE is further complicated by a dramatic redistribution of pulmonary blood flow accompanied by an equally dramatic reduction in right ventricular afterload. The severity of reperfusion pulmonary oedema is highly variable, ranging from a mild form resulting in postoperative hypoxaemia in most affected patients to profound alveolar haemorrhage that may be fatal. In our experience no patient died of reperfusion pulmonary oedema. Careful management of ventilation and fluid balance will minimise alveolar capillary leak.

\section{LONG TERM OUTCOME}

The reported mid term and long term mortality rates vary, at least in part, according to the different periods in which the surgery was performed. In general results have gradually improved with practice, except for those of the San Diego Medical Center which were good even at the beginning of the 1990s. s. $^{3-12} 13$ Another factor which may account for different results is that follow up studies may extend for more than a few years, during which time pulmonary hypertension may recur after an asymptomatic period in patients in whom the underlying cause persists. In our three year follow up, during which regular anticoagulant treatment was administered, four patients of 27 relapsed into severe pulmonary hypertension and died from right ventricular insufficiency. Two patients had a history of venous thromboemobolism associated with antiphospholipid antibody syndrome. A similar rate of relapse was reported by the San Diego Medical Center. Lifelong anticoagulant treatment is strongly recommended after PTE. Recurrent thromboembolism necessitating a second thromboendarterectomy has occurred in several patients in whom anticoagulant treatment was discontinued or maintained at a subtherapeutic level. ${ }^{14}$

The first long term follow up studies of PTE have shown sustained improvements in pulmonary haemodynamics and gas exchange. ${ }^{12}{ }^{13}$ An interesting cross sectional study by Archibald and colleagues addressed the long term effects of this operation on functional status, survival, and quality of life in a large sample of patients who had undergone PTE surgery at the University of California, San Diego; they found that most patients had improved substantially, with minimal disease related health care utilisation about three years after the PTE. ${ }^{8}$ In our follow up of patients treated with PTE since 1994 we have observed that only a few patients did not have a satisfactory recovery, either because of operative failure, hypertension relapse, or the effect of preoperative hypertension on vessels in non-obstructed segments of the lungs, including medial and intimal hypertrophy and obliteration. The majority of the patients, even the most compromised ones, had excellent long lasting results. Most of the improvement in clinical and cardiopulmonary function parameters was achieved in the first three months as a result of the relief of central mechanical obstruction. We observed further improvement at one, two, and three years in exercise tolerance; in addition to the gradual improvement in the ventilation/perfusion ratio this was probably caused by the change in overall clinical status which allowed progressive training.

\section{SUMMARY}

The success of PTE has now been confirmed, particularly by the recent results on long term effects, and it remains the primary treatment for CTEHP. Although PTE is so successful that alternative approaches to CTEPH may seem heretical, other interventional strategies such as balloon pulmonary angioplasty should be implemented for selected patients - that is, those with surgically inaccessible disease or comorbid illness.

Unfortunately, we believe that Jamieson's statement about under recognition of the disease and the lack of confidence in PTE (even among cardiac surgeons) still holds true. Thus, we completely agree with his last recommendation that physicians should be encouraged to identify patients with this now curable disease.

\section{Authors' affiliations}

I Cerveri, Division of Respiratory Diseases, IRCCS Policlinico San Matteo, University of Pavia, Pavia, Italy

A M D'Armini, M Viganò, Department of Surgery and Organ

Transplantation - Division of Cardiac Surgery, IRCCS Policlinico San

Matteo University of Pavia, Italy

\section{REFERENCES}

1 Fedullo PF, Rubin L, Kerr KM, et al. The natural history of acute and chronic thromboembolic disease: search for the missing link. Eur Respir J 2000;15:435-37.

2 Jamieson SW. Pulmonary thromboendarterectomy. Hear 1998;79:118-20.

3 Fedullo PF, Auger WR, Kerr KM, et al. Chronic thromboembolic pulmonary hypertension. N Engl J Med 2001;345: 1465-72.

4 Zoia MC, D'Armini AM, Beccaria M, et al. on behalf of the Pavia Thromboendarterectomy Group. Mid term effects of pulmonary thromboendarterectomy on clinical and cardiopulmonary function status. Thorax 2000;57:608-12.

5 Jamieson SW, Auger WR, Fedullo PF, et al. Experience and results of 150 pulmonary thromboendarterectomy operations over a 29 month period. J Thorac Cardiovasc Surg 1993;106:1 16-27.

6 D'Armini AM, Cattadori B, Monterosso C, et al. Pulmonary thromboendarterectomy in patients with chronic thromboembolic pulmonary hypertension: hemodynamic characteristics and changes. Eur J Cardiothorac Surg 2000;18:696-702.

7 D'Armini AM, Cattadori B, Monterosso C, et al. Surgical therapy for chronic thromboembolic pulmonary hypertension: criteria for choosing lung transplant vs thromboendarterectomy. J Heart Lung Transplant 2001;20:218.

8 Archibald CJ, Auger WR, Fedullo PF, et al. Long-term outcome after pulmonary thromboendarterectomy. Am J Respir Crit Care Med 1999:160:523-8

9 Feinstein JA, Goldhaber SZ, Lock JE, et al. Balloon pulmonary angioplasty for treatment of chronic thromboembolic pulmonary hypertension. Circulation 2001;103:10-13.

10 Daily PO, Dembitsky WP, Jamieson SW. The evaluation and the current state of the art of pulmonary thromboendarterectomy. Semin Thorac Cardiovasc Surg 1999;11:152-63.

11 Jamieson SW, Kapelanski DP. Pulmonary endoarterectomy. Curr Probl Surg 2000;37:165-252

12 Kramm T, Mayer E, Dahm M, et al. Long-term results after thromboendarterectomy for chronic pulmonary embolism. Eur J Cardiothorac Surg 1999:15:579-84.

13 Tanabe N, Okada O, Nakagawa Y, et al. The efficacy of pulmonary thromboendarterectomy on long-term gas exchange. Eur Repir $J$ 1997; 10:2066-72

14 Mo M, Kapelanski DP, Mitruka SN, et al. Reoperative pulmonary thromboendarterectomy. Ann Thorac Surg 1999;68:1770-6. 